Revista Brasileira de Agricultura Irrigada v.8, nº. 3, p. 220 - 233, 2014

ISSN 1982-7679 (On-line)

Fortaleza, CE, INOVAGRI - http://www.inovagri.org.br

DOI: $10.7127 /$ rbai.v8n300214

Protocolo 214/14 - 15/01/2014 Aprovado em 26/04/2014

\title{
CURVA CARACTERÍSTICA DA VAZÃO DE GOTEJADORES EM DIFERENTES TEMPERATURAS DA ÁGUA E AÇÃO DO CLORO OU ÁCIDO
}

\author{
Marconi Batista Teixeira ${ }^{1}$, Rubens Duarte Coelho $^{2}$, Fernando Nobre Cunha ${ }^{3}$, Nelmício \\ Furtado da Silva $^{4}$
}

\begin{abstract}
RESUMO
O objetivo foi desenvolvido visando quantificar possíveis distúrbios de vazão em emissores do tipo gotejador submetidos à aplicação de cloro livre e acidificação em condições estática e dinâmica de fluxo de água no sistema. O experimento foi realizado utilizando-se 14 modelos de gotejadores autocompensantes com suas respectivas linhas gotejadoras montadas em uma bancada de ensaios em estrutura metálica. Os tratamentos utilizados no experimento foram: a) aplicação dinâmica de $100 \mathrm{mg} \mathrm{L}^{-1}$ de cloro livre com pH na faixa de 5,5 a 6,0; b) aplicação estática de $100 \mathrm{mg} \mathrm{L}^{-1}$ de cloro livre com pH na faixa de 5,5 a 6,0; c) aplicação de ácido nítrico com $\mathrm{pH}$ na faixa de 2,0 a 3,0 durante $1 / 2 \mathrm{~h}$ (sistema pressurizado). Foram traçadas curvas vazão versus pressão para cada tratamento em três diferentes temperaturas da água (15, 25 e $40^{\circ} \mathrm{C}$ ). $\mathrm{O}$ delineamento experimental utilizado foi o inteiramente aleatorizado em esquema fatorial, com utilização dos testes " $F$ " para análise de variância e Tukey, a 5\% de significância, para comparação de médias e análise de regressão. Os resultados obtidos mostram que para o tratamento da água com $100 \mathrm{mg} \mathrm{L}^{-1}$ de cloro livre em condição dinâmica, ocorreu um decréscimo de 10 a $20 \%$ em média da vazão $\left(\mathrm{L} \mathrm{h}^{-1}\right)$ para alguns modelos de gotejadores mais sensíveis aos produtos químicos, sendo que o modelo I apresentou redução drástica de vazão na ordem de $100 \%$ em virtude do bloqueio total do labirinto do emissor, após $2688 \mathrm{~h}$ de aplicação contínua de cloro. Os tratamentos estáticos não apresentaram variações acentuadas de vazão média $\left(\mathrm{L} \mathrm{h}^{-1}\right)$. Verificou-se relação linear entre a vazão e a temperatura da água para os gotejadores novos, sendo que após a aplicação dos diferentes tratamentos, as curvas vazão versus pressão para cada modelo de gotejador sofreram influência tanto da pressão quanto da temperatura de maneira distinta.
\end{abstract}

Palavras-chave: cloração, acidificação, temperatura, emissores autocompensantes.

\footnotetext{
1 Eng. Agrônomo, Prof. Dr. Instituto Federal Goiano - IFGoiano - Rio Verde, GO, email: marconibt@gmail.com

${ }^{2}$ Eng. Agrônomo, Prof. Livre Docente, DER - Departamento de Engenharia Rural, Escola Superior de Agricultura Luiz de Queiroz, Universidade de São Paulo, Piracicaba, SP, C.P.: 9, CEP: 13418-900, fone (0xx19) 3447-8551, email: rdcoelho@esalq.usp.br

${ }^{3}$ Eng. Agrônomo, Mestrando em Ciências Agrárias - Agronomia, IFGoiano - Câmpus Rio Verde, Rio Verde, GO, email: fernandonobrecunha@ hotmail.com

${ }^{4}$ Eng. Agrônomo, Mestrando em Ciências Agrárias - Agronomia, IFGoiano - Câmpus Rio Verde, Rio Verde, GO, email: nelmiciofurtado@gmail.com
} 


\title{
FLOW RATE DRIPPERS EXPOSED TO THE ACTION OF CHLORINE OR NITRIC ACID AT DIFFERENT WATER TEMPERATURES
}

\begin{abstract}
The objective was developed to quantify possible flow rate variation in emitters (drip irrigation) submitted to the application of free chlorine and acidification under static and dynamics conditions of water flow. The experiment was accomplished with 14 models of pressure compensating emitters set up in a metallic bench. The treatments used in the experiment were the following ones: a) dynamic application of $100 \mathrm{mg} \mathrm{L}^{-1}$ of free chlorine with $\mathrm{pH} 5,5$ to 6,$0 ; \mathrm{b}$ ) static application of $100 \mathrm{mg} \mathrm{L}^{-1}$ of free chlorine with $\mathrm{pH} 5,5$ to 6,0 ; c) application of acid nitric with $\mathrm{pH} 2,0$ to 3,0 during $1 / 2 \mathrm{~h}$ (pressurized system). The operating pressures and emitter model combination were: $50,100,200,300,400 \mathrm{kPa}$ for emitters A to $\mathrm{N}$, in three different water temperatures $\left(15,25\right.$ and $\left.40{ }^{\circ} \mathrm{C}\right)$. The statistical analysis of the treatments were accomplished by Tukey test at $5 \%$ probability. The obtained results show that for the treatment of the water with $100 \mathrm{mg} \mathrm{L}^{-1}$ of free chlorine in dynamic condition, a reduction from 10 to $20 \%$ of the flow rate $\left(\mathrm{L} \mathrm{h}^{-1}\right)$ occurred for some models more sensitive to chemical products. Model I presented drastic reduction of flow rate in the order of $100 \%$ by total blockade of the labyrinth of the emitter, after $2688 \mathrm{~h}$ of continuous application of $100 \mathrm{mg}$ $\mathrm{L}^{-1}$ of chlorine free with $\mathrm{pH} 5,5$ to 6,0 . The static treatments didn't present accentuated variations of medium flow rate $\left(\mathrm{L} \mathrm{h}^{-1}\right)$. For pressure compensating emitters, where the discharge sensitivity to temperature is insignificant, after the application of the different treatments, the measured discharge/pressure relationship for each emitter model was sensitive to pressure as well to temperature.
\end{abstract}

Keywords: chlorination, acidification, temperature, pressure compensating emitters.

\section{INTRODUÇÃO}

Em regiões onde a precipitação pluvial não é suficiente para atender às necessidades hídricas das culturas, ou mesmo onde ela é suficiente, mas irregularmente distribuída ao longo do ano, a aplicação da irrigação torna-se necessária ao pleno desenvolvimento das espécies cultivadas.

A irrigação localizada por gotejamento possibilita manter a umidade do solo na zona radicular próximo da capacidade de campo, com a aplicação de pequenas vazões em elevada frequência, aplicações de fertilizantes via água de irrigação, baixas pressões e altas eficiências, geralmente superior a $90 \%$, possibilitando um controle eficiente da lâmina de irrigação (BERNARDO et al., 2006).
A irrigação localizada está sujeita a alterações de vazão, tendo como consequência na sua pior hipótese a obstrução da emissão, pelo fato de utilizar emissores com orifícios de passagem de água muito pequenos, esta limitação acontece principalmente na irrigação por gotejamento que possui como grande limitação o fato de seus emissores possuírem orifícios e labirintos pequenos, suscetíveis ao entupimento, ocasionando perda da uniformidade de irrigação ao longo do tempo, porém este fato pode ser agravado com a utilização da aplicação de nutrientes via água de irrigação assim como efluentes com altos teores de sólidos e bactérias formadoras de biofilme (CUNHA et al., 2013). 
A cloração é o método mais utilizado para controlar o entupimento de origem biológica, sendo que a aplicação pode ser feita de forma contínua ou intermitente. A aplicação contínua é feita utilizando-se baixas dosagens de cloro (1 a $10 \mathrm{mg} \mathrm{L}^{-1}$ de cloro livre), e a intermitente pode ser efetuada utilizando-se aplicações de choque, fazendo uso de altas dosagens de cloro (acima de $100 \mathrm{mg} \mathrm{L} \mathrm{L}^{-1}$ ). O tratamento com utilização combinada de ácido e cloro apresenta vantagens, em relação ao uso exclusivo de ácidos ou cloro, tanto em termos de efetividade como também de economicidade, sendo que o tratamento com ácido é normalmente efetuado com freqüência semanal, utilizando-se o mesmo tempo da aplicação do cloro (NAKAYAMA e BUCKS, 1991).

Objetivou-se com o presente trabalho quantificar possíveis distúrbios de vazão em emissores do tipo gotejador (irrigação localizada) submetidos à aplicação de cloro livre e acidificação em condições estática e dinâmica de fluxo de água no sistema.

\section{MATERIAL E MÉTODOS}

O experimento foi realizado no Laboratório de Irrigação do Departamento de Engenharia de Biossistemas da Escola Superior de Agricultura "Luiz de Queiroz" ESALQ/USP, utilizando-se uma bancada de ensaios em estrutura metálica, com 11,0 m de comprimento por 4,0 m de largura e 5,80 m de altura.

Em cada nível foi realizado um tratamento específico: Nível 1: Curvas vazão em função da pressão em diferentes temperaturas da água $\left(15,25\right.$ e $\left.40^{\circ} \mathrm{C}\right)$ após 2688 horas de ensaio utilizando aplicação contínua de $100 \mathrm{mg} \mathrm{L}^{-1} \mathrm{de}$ cloro livre (hipoclorito de sódio a 12\%) com $\mathrm{pH}$ na faixa de 5,5 a 6,0 durante $24 \mathrm{~h} \mathrm{dia}^{-1}$; Nível 2: Curvas vazão em função da pressão em diferentes temperaturas da água $\left(15,25\right.$ e $\left.40^{\circ} \mathrm{C}\right)$ após 2688 horas de ensaio utilizando aplicação intermitente de $100 \mathrm{mg} \mathrm{L}^{-1}$ de cloro livre (hipoclorito de sódio a $12 \%$ ) com $\mathrm{pH}$ na faixa de 5,5 a 6,0 durante $1 / 2 \mathrm{~h}+1$ semana de descanso da solução na linha lateral; Nível 3: Curvas vazão em função da pressão em diferentes temperaturas da água $\left(15,25\right.$ e $\left.40^{\circ} \mathrm{C}\right)$ após 2688 horas de ensaio utilizando aplicação intermitente de ácido nítrico com $\mathrm{pH}$ na faixa de 2,0 a 3,0 durante $1 / 2 h+1$ semana de descanso da solução na linha lateral.

Foram realizadas curvas vazão em função da pressão para 14 modelos de gotejadores autocompensantes instalados em cada nível, utilizando os seguintes valores de pressão e temperatura da água: Pressão: 50, 100, 200, 300, e $400 \mathrm{kPa}$; Temperatura da água: $15^{\circ} \mathrm{C}\left(15^{\circ} \mathrm{C} \pm\right.$ $\left.4{ }^{\circ} \mathrm{C}\right), 25{ }^{\circ} \mathrm{C}\left(25{ }^{\circ} \mathrm{C} \pm 3{ }^{\circ} \mathrm{C}\right)$ e $40^{\circ} \mathrm{C}\left(40{ }^{\circ} \mathrm{C} \pm 1\right.$ $\left.{ }^{\mathrm{o}} \mathrm{C}\right)$.

Foram instalados 14 modelos de gotejadores autocompensantes em cada nível, com vazões e espaçamentos diferentes, ou seja, o número de gotejador presente na linha dependeu do espaçamento fornecido pelo fabricante, 
porém foi assegurado um número mínimo de 10 emissores para cada modelo analisado.

Em cada ensaio com um determinado modelo de gotejador, envolvendo uma determinada dosagem de cloro ou ácido nítrico, $\mathrm{pH}$, temperatura e tempo de exposição, foi utilizada uma linha de gotejadores novos, para evitar a influência de um determinado tratamento sobre outro, permitindo, assim, verificar adequadamente como ocorreu o dano químico na membrana de compensação do modelo de gotejador avaliado.

$\mathrm{Na}$ Tabela 1 encontra-se a listagem dos gotejadores que foram utilizados na pesquisa, com suas características técnicas e a vazão mais comercializada no mercado nacional.

Tabela 1. Características técnicas dos modelos com fluxo autocompensante, vazões, diâmetro nominal $(\varnothing \mathrm{N})$ e pressão de serviço $(\mathrm{kPa})$.

\begin{tabular}{|c|c|c|c|c|}
\hline Fabricante & Modelo & Vazão & $\varnothing \mathrm{N}$ & $\begin{array}{c}\text { Pressão } \\
\text { de serviço }\end{array}$ \\
\hline & & $\mathrm{L} \mathrm{h}^{-1}$ & $\mathrm{~mm}$ & $\mathrm{kPa}$ \\
\hline Irrimon & Irridrip & 2,5 & 16 & $100-350$ \\
\hline Carborundum & Carbodrip PC & 2,3 & 16 & $100-400$ \\
\hline Netafim & Uniram & 1,6 & 17 & $50-400$ \\
\hline Naan & Naan PC & 3,8 & 16 & $100-350$ \\
\hline Plastro & Hydro PCND & 2,35 & 16 & $80-350$ \\
\hline Netafim & Ram & 2,3 & 17 & $50-400$ \\
\hline Drip In & Drip In PC & 2,4 & 16 & $100-350$ \\
\hline Naan & Naan PC & 2,1 & 16 & $100-350$ \\
\hline Netafim & Ram & 1,6 & 16 & $50-400$ \\
\hline Irrimon & Twin Plus & 1,8 & 17,5 & $100-350$ \\
\hline Plastro & Hydro PC & 2,2 & 16 & $80-350$ \\
\hline Netafim & Dripnet PC & 1,6 & 17 & $50-400$ \\
\hline Plastro & Hydro PC & 2,0 & 17 & $80-350$ \\
\hline Irrimon & Vip Line & 3,6 & 16 & $100-350$ \\
\hline
\end{tabular}

Fonte: Catálogos dos fabricantes.
Como os gotejadores ensaiados são produtos comerciais, os dados e análises aqui apresentadas foram codificados para evitar qualquer tipo de especulação comercial dos resultados apresentados, uma vez que os ensaios conduzidos não são normatizados. As letras utilizadas na codificação dos modelos de emissores $(\mathrm{A}, \mathrm{B}, \mathrm{C}, \ldots, \mathrm{N})$, não têm nenhuma relação com os nomes comerciais dos produtos e nem com a sequência de emissores apresentada na Tabela 1. A identificação parcial dos gotejadores poderá ser solicitada diretamente aos autores, através dos e-mails descritos na identificação dos mesmos.

A água que foi utilizada nos ensaios foi proveniente da estação de tratamento de água da ESALQ/USP- Piracicaba, SP; após passar pelos gotejadores, era recolhida por um sistema de calhas que a reconduzia ao tanque de captação, constituindo, assim, um sistema de recirculação de água.

Foi utilizado um filtro de disco de 100 mesh para a retirada de partículas sólidas que porventura entravam no sistema. Utilizou-se um registro de 2" na linha principal e um registro de 3/4" na entrada de cada nível. As linhas gotejadoras foram unidas no início e ao final por registros de 1/2" e conexões de PVC, sendo sustentadas por dispositivos em estrutura metálica.

O sistema de motobomba utilizado nos ensaios foi composto por duas bombas centrífugas marca KSB, modelo KSB Megabloc 32-160.1R (utilizada para aplicação de água 
em todo o sistema e lavagem das linhas laterais) e KSB Hydrobloc C 1000 (aplicação dos tratamentos químicos). A operacionalização do funcionamento da bancada, quanto ao horário de início e de parada, foi efetuada manualmente, obedecendo rigorosamente os horários de aplicação e leitura.

À entrada de cada nível do térreo da bancada, foi instalada uma tomada de pressão, permitindo o ajuste da pressão a cada medição de vazão, e se necessário, ajustada àquela preestabelecida. Para a medição da pressão de serviço, à entrada da linha lateral, foi utilizado um manômetro digital com faixa de leitura de 0 - $700 \mathrm{kPa}$, abaixo segue a descrição dos níveis de 1 ao 3 .

Nível 1 (aplicação contínua de $100 \mathrm{mg}$ $\mathrm{L}^{-1}$ de cloro livre com $\mathrm{pH}$ na faixa de 5,5 a 6,0 ): a aplicação do tratamento químico da água utilizando $100 \mathrm{mg} \mathrm{L}^{-1}$ de cloro livre era realizada em um intervalo de $24 \mathrm{~h}$ utilizando-se pressão de $200 \mathrm{kPa}$, temperatura da solução igual a $25^{\circ} \mathrm{C} \pm$ $3{ }^{\circ} \mathrm{C}$ e manutenção do $\mathrm{pH}$ na faixa de 5,5 a 6,0 . Para a obtenção da calda contendo $100 \mathrm{mg} \mathrm{L}^{-1}$ de cloro livre fez-se uma curva de titulação utilizando-se hipoclorito de sódio (12\%) e ácido fosfórico $85 \%$ P.A.

Após 2688 horas de ensaio utilizando o tratamento descrito acima, foram realizadas as curvas vazão em função da pressão em diferentes temperaturas da água para os 14 modelos de gotejadores ensaiados.
O procedimento para as leituras de vazão de cada gotejador consistiu da pressurização do sistema $(200 \mathrm{kPa})$, posicionamento de recipientes plásticos (com dimensões adequadas para receber um volume de até $1 \mathrm{~L})$ sob os respectivos gotejadores. Os coletores foram posicionados sob os respectivos gotejadores com uma defasagem de 5 segundos, com retirada sequencial dos mesmos após 10 minutos e defasagem de 5 segundos e transporte à bancada de pesagem, sendo feito em seguida a pesagem e tabulação dos dados. Para a confecção das curvas o tempo de coleta foi de 5 $\min$.

Para se obter maior exatidão foi utilizado o método gravimétrico para a determinação do volume coletado de cada emissor, expressandose os valores de vazão em $\mathrm{L} \mathrm{h}^{-1}$. Foi utilizada uma balança de precisão certificada (OHAUS) com precisão de $0,01 \mathrm{~g}$. Cada coletor com água foi pesado; descontando-se sua tara.

Depois de tabulados os pesos, efetuaram-se os cálculos da vazão em cada faixa de pressão, pela eq. (1).

$$
q=\frac{P}{1000 t} 60
$$

em que:

q - vazão do gotejador (após tratamento químico), $\mathrm{L} \mathrm{h}^{-1}$;

$\mathrm{P}$ - peso da água coletada, $\mathrm{g}$;

$\mathrm{t}$ - tempo de coleta, min; 
Nível 2 (aplicação de $100 \mathrm{mg} \mathrm{L}^{-1}$ de cloro livre por $1 / 2 \mathrm{~h}$ e descanso da solução na linha por 1 semana): as linhas gotejadoras foram inseridas dentro de tubos PVC com diâmetro nominal de $32 \mathrm{~mm}$ e vedadas junto aos registros no início e no final com o uso de fita isolante de alta fusão, visando manter a solução de $100 \mathrm{mg} \mathrm{L}^{-1}$ de cloro livre em contato com toda a parede interna e externa da mangueira e do gotejador.

Para realizar-se a leitura de vazão das linhas gotejadoras, após 1 semana de aplicação, utilizou-se o medidor magnético Conaut Krohne modelo IFS 4000 W/6 com conversor de sinal modelo IFC $090 \mathrm{D}$ e com faixa de leitura de $0-90 \mathrm{~m}^{3} \mathrm{~h}^{-1}$ e precisão inferior a $1 \%$ de erro para a leitura de vazão das linhas gotejadoras.

Esta mesma solução após a aplicação no Nível 2 era utilizada para a aplicação dinâmica no Nível 1 (tratamento de choque com aplicação dinâmica de $100 \mathrm{mg} \mathrm{L}^{-1}$ de cloro livre, com pH na faixa de 5,5 a 6,0), evitando assim, desperdício de solução. Para a manutenção do $\mathrm{pH}$ na faixa de 5,5 a 6,0 utilizou-se ácido fosfórico $85 \%$ P.A.

O procedimento para a leitura de vazão da linha gotejadora utilizando-se o medidor magnético, consistiu da pressurização do sistema com estabilização da pressão em $200 \mathrm{kPa}$ no início de cada linha, utilizando um manômetro de Bourdon, e aplicação da solução num intervalo de $1 / 2$ hora. O sistema de aplicação permitia a recirculação da solução aplicada conforme o seguinte esquema: reservatório $\rightarrow$ gotejadores $\rightarrow$ telha $\rightarrow$ calha $\rightarrow$ tubo de PVC $100 \mathrm{~mm} \rightarrow$ reservatório. Após a leitura realizava-se a leitura com o registro fechado para observar a vazão residual. A leitura de vazão no medidor foi feita $\mathrm{em} \mathrm{m}^{3} \mathrm{~h}^{-1}$, conforme a equação 2, sendo que para cada leitura aguardava-se a estabilização do medidor magnético de vazão.

Devido às linhas gotejadoras estarem envolvidas individualmente por tubos de PVC 32 $\mathrm{mm}$, foram feitos furos no tubo na parte superior correspondente ao centro da mangueira. Estes furos permitiam a recirculação da solução e que ao estabilizar a pressão de funcionamento do sistema motobomba, não ocorresse vazamento junto às emendas com fita isolante de alta fusão feitas nas extremidades das mangueiras próximas dos registros, evitando também a perda de solução após o encerramento da aplicação. Ao final da aplicação os registros eram fechados com o sistema ainda em funcionamento, para evitar retorno da solução para o reservatório, garantindo assim que a solução permanecesse em contato com toda a superfície interna e externa da linha gotejadora. Para evitar uma perda acentuada da solução ao longo da semana, foram colocadas cortinas laterais plásticas na bancada de ensaio.

A eq. (2) mostra como foi feito o cálculo da vazão média $\left(\mathrm{L} \mathrm{h}^{-1}\right)$ de cada modelo de gotejador ensaiado, a partir da vazão total $\left(\mathrm{m}^{3}\right.$ $\mathrm{h}^{-1)}$ obtida pelo uso do medidor magnético de vazão. 


$$
q_{m}=\frac{Q}{1000 N}
$$

em que:

$$
\begin{aligned}
& q_{m} \text { - vazão média do gotejador, } \mathrm{L} \mathrm{h}^{-1} ; \\
& Q \text { - vazão total, } \mathrm{m}^{3} \mathrm{~h}^{-1} ; \mathrm{e} \\
& N \text { - número de emissores na linha gotejadora. }
\end{aligned}
$$

Nível 3 (aplicação de ácido nítrico com pH na faixa de 2,0 a 3,0 por $1 / 2 \mathrm{~h}$ e descanso da solução na linha por 1 semana): no Nível 3 as linhas gotejadoras foram envolvidas por tubo PVC com diâmetro nominal de $25 \mathrm{~mm}$ e vedadas junto aos registros no início e no final com o uso de fita isolante de alta fusão, para manter a solução de ácido nítrico com pH na faixa de 2,0 a 3,0 em contato com toda a parede interna e externa da mangueira e do gotejador, sendo também feitos furos na parte superior do tubo correspondente ao centro da mangueira.

Utilizou-se o mesmo procedimento adotado para o Nível 2 com aplicação estática de $100 \mathrm{mg} \mathrm{L}^{-1}$ de cloro livre por $1 / 2 \mathrm{~h}$ e descanso na linha gotejadora por 1 semana, sendo que preparava-se uma calda da solução de ácido nítrico com $\mathrm{pH}$ na faixa de 2,0 a 3,0 em um volume de água correspondente a $150 \mathrm{~L}$, no reservatório. Em seguida, realizava-se a leitura do $\mathrm{pH}$ e da temperatura da solução.
Características hidráulicas dos emissores sob diferentes temperaturas da água: foram feitas curvas vazão versus pressão para os tratamentos com aplicação dinâmica e estática de $100 \mathrm{mg} \mathrm{L}^{-1}$ de cloro livre (Nível 1 e 2) e aplicação estática de ácido nítrico $(\mathrm{pH} 2,0)$ por $1 / 2$ hora. Sendo que para a confecção destas curvas utilizou-se as pressões de 50, 100, 200, 300 e $400 \mathrm{kPa}$.

Para cada uma das pressões descritas acima, foram realizados ensaios com as seguintes temperaturas da água: $15^{\circ} \mathrm{C}(10$ a $\left.15^{\circ} \mathrm{C}\right), 25^{\circ} \mathrm{C}\left(25^{\circ} \mathrm{C} \pm 3^{\circ} \mathrm{C}\right)$, e $40^{\circ} \mathrm{C}\left(40^{\circ} \mathrm{C} \pm 1^{\circ} \mathrm{C}\right)$.

Com relação à avaliação das linhas gotejadoras na temperatura de $15{ }^{\circ} \mathrm{C}$, a melhor alternativa encontrada, foi trabalhar com uma faixa de temperatura de 10 a $15^{\circ} \mathrm{C}$ e o uso de gelo para auxiliar o freezer na manutenção da água dentro desta faixa.

A diferença de temperatura entre o freezer e o final da linha lateral encontrada, foi no máximo de $1^{\circ} \mathrm{C}$. Para tanto, o procedimento seguido diariamente para a avaliação das linhas gotejadoras foi a seguinte: media-se a temperatura da água dentro do freezer, caso estivesse bem abaixo da faixa utilizada nas análises, procedia-se à pressurização do sistema $(200 \mathrm{kPa})$ com todos os registros abertos para recircular a água até atingir a faixa desejada e também recirculava a água com a utilização de um retorno da bomba direto para o freezer (este 
retorno também tinha a função de auxiliar no controle da pressão durante os ensaios), sendo no entanto medida a temperatura da água tanto no freezer, quanto no primeiro gotejador da primeira linha a ser avaliada. Fechava-se o registro principal (1 $1 / 2$ ") e todos os registros tanto no início quanto no final, com exceção da primeira linha a ser avaliada que permanecia com ambos os registros abertos.

Cuidadosamente a pressão do sistema era ajustada para a pressão requerida e procedia-se a medição da temperatura no primeiro e último gotejador da linha em análise e coletavam-se as vazões de todos os gotejadores na linha utilizando-se o método gravimétrico. Este procedimento foi o mesmo utilizado para a avaliação de todas as demais linhas gotejadoras. Assim, que a temperatura ultrapassava os $15^{\circ} \mathrm{C}$, o sistema era desligado e aguardava-se o resfriamento da água dentro do freezer novamente até atingir $10{ }^{\circ} \mathrm{C}$, utilizando-se no entanto gelo para acelerar o processo.

Para aquecer a água, foi utilizado um conjunto de resistências no reservatório (tratamento). E para permitir o monitoramento do nível de aquecimento da água, devido o uso das resistências e a recirculação da água no sistema, foi utilizado um sistema de controle automático instalado junto ao reservatório para monitoramento, evitando assim, temperaturas acima do desejado. A diferença observada foi de no máximo $\Delta \mathrm{T}=1{ }^{\circ} \mathrm{C}$ durante todo o período do ensaio.
Foi adotado o delineamento inteiramente casualizado, em esquema fatorial, com utilização dos testes "F" para análise de variância e Tukey, a 5\% de significância, para comparação de médias e análise de regressão. As análises estatísticas foram realizadas utilizando-se o programa SAS (1999), pelo procedimento GLM. A análise de regressão foi feita para as variáveis que apresentaram diferença significativa a 1 ou 5\%. A repetição foi dada pelo número mínimo de gotejadores presentes na linha lateral (dez gotejadores), e não pelo número de linhas.

\section{RESULTADOS E DISCUSSÃO}

Observa-se uma variação dos valores de vazão média e vazão relativa após cada leitura semanal, podendo de uma semana para outra a vazão média e a vazão relativa apresentar valores baixos e altos respectivamente. Isto ocorre porque apesar do medidor magnético de vazão apresentar precisão de $99 \%$, a leitura da vazão foi feita após a estabilização do aparelho e da pressão na linha, mas a ocorrência de pequenas alterações de pressão na linha e também variações quanto à leitura da vazão residual da linha lateral poderiam contribuir para estas diferenças de leitura de vazão. No entanto, esta diferença não foi superior a $7 \%$ da leitura de vazão da linha de uma semana para outra, para 
nenhum dos modelos de emissores ensaiados. A vazão real da linha gotejadora durante todo o ensaio foi obtida da subtração da vazão da linha medida com o registro aberto no início da vazão residual (leitura da vazão da linha com o registro no início da linha fechado).

Para todos os emissores avaliados, aplicação estática de ácido nítrico com pH na faixa de 2,0 a 3,0 por $1 / 2$ h e descanso da solução na linha por 1 semana, não resultou em danos à membrana de compensação, pois a influência nas curvas dos emissores foi mínima.

As Figuras 1 a 7 mostram as curvas vazão versus pressão para cada modelo de gotejador comparando os diferentes tratamentos químicos para a temperatura da água igual a 15 , 25 e $40^{\circ} \mathrm{C}$, utilizando as pressões de: 50, 100, 200, 300 e $400 \mathrm{kPa}$. A nomenclatura utilizada para identificação dos tratamentos foi a seguinte: SA - Sem aplicação (testemunha), 100 AD Aplicação dinâmica de 100 mg L ${ }^{-1}$ de cloro livre, $100 \mathrm{AE}$ - Aplicação estática de $100 \mathrm{mg} \mathrm{L}^{-1}$ de cloro livre, e pH 2,0 AE - Aplicação estática de ácido nítrico a $\mathrm{pH} 2,0$ por $1 / 2 \mathrm{~h}$.

Os números de horas correspondentes a cada tratamento são: Testemunha $(0 \mathrm{~h} \quad-$ gotejador novo), aplicação dinâmica de cloro (2688 h com exceção dos modelos A e N com 1512 h), aplicação estática de cloro (3192 h com exceção dos modelos A e N com 1344 h) e aplicação estática de ácido nítrico por $1 / 2 \mathrm{~h}$ (2688 h com exceção dos modelos A e N com $1344 \mathrm{~h})$.

As curvas vazão para os modelos A, B, C, G, H, M e N não são apresentadas por terem apresentado comportamento distinto, com redução da vazão em relação à testemunha mais evidenciado para os tratamentos com cloro, independentemente da temperatura da água.

A $15^{\circ} \mathrm{C}$ a aplicação de $100 \mathrm{AD}$ provocou quedas na vazão de $50 \%$ na pressão de $50 \mathrm{kPa}$ para o modelo D, sendo essa redução decrescente com o aumento da pressão, desta forma na pressão de $400 \mathrm{kPa}$ a vazão mostrou uma diminuição de $26 \%$, já para os tratamentos SA, 100AE e pH2,0AE observou-se diferenças pequenas na vazão de no máximo 6\%; comportamento similar a este foi verificado na temperatura de $40^{\circ} \mathrm{C}$, com diferença na vazão do tratamento 100AD para os demais de $37 \%(50 \mathrm{kPa})$ e de $19 \%$ (400 kPa), entretanto o mesmo não foi observado na temperatura de $25^{\circ} \mathrm{C}$, onde a vazão no tratamento 100AD manteve-se praticamente constante $\left(1 \mathrm{~L} \mathrm{~h}^{-1}\right)$, ocorrendo o mesmo com as diferenças, que foram de 38 (tratamento SA e $\mathrm{pH} 2,0 \mathrm{AE})$ e $12 \%$ (tratamento 100AE). Os melhores resultados foram obtidos com a dose de hipoclorito de $150 \mathrm{mg} \mathrm{L}^{-1}$ em quatro aplicações. 

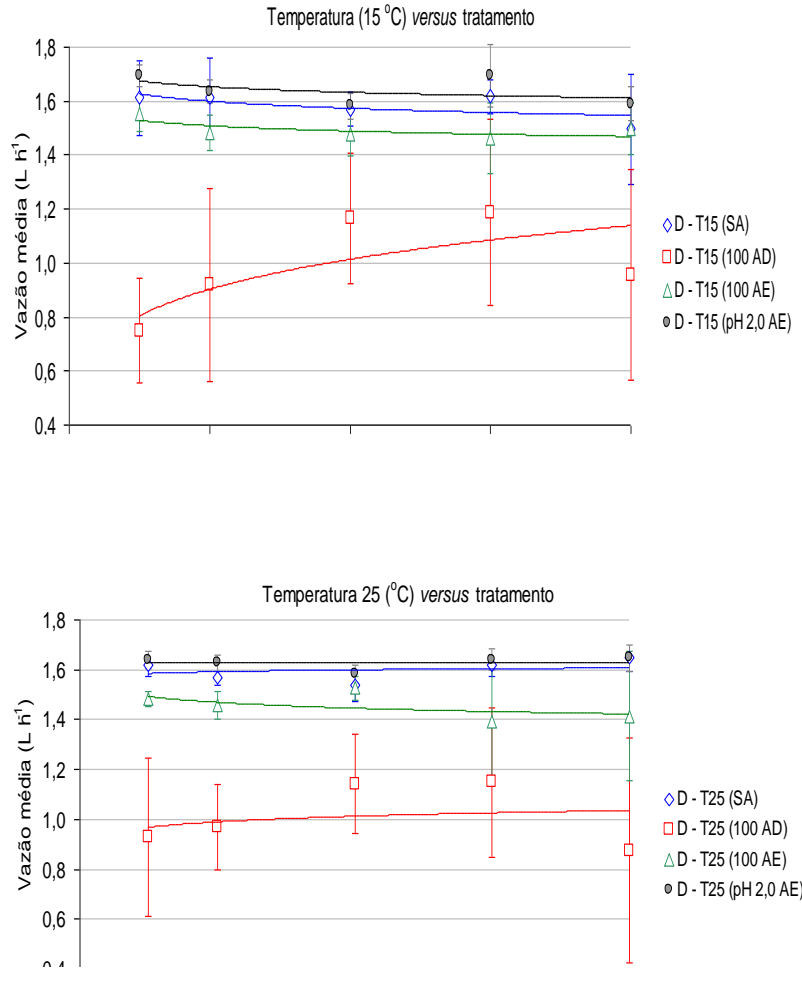

$\diamond \mathrm{D}-\mathrm{T} 25(\mathrm{SA})$

$\square \mathrm{D}-\mathrm{T} 25(100 \mathrm{AD})$

$\triangle \mathrm{D}-\mathrm{T} 25(100 \mathrm{AE})$

OD - T25 (pH2,0 AE)

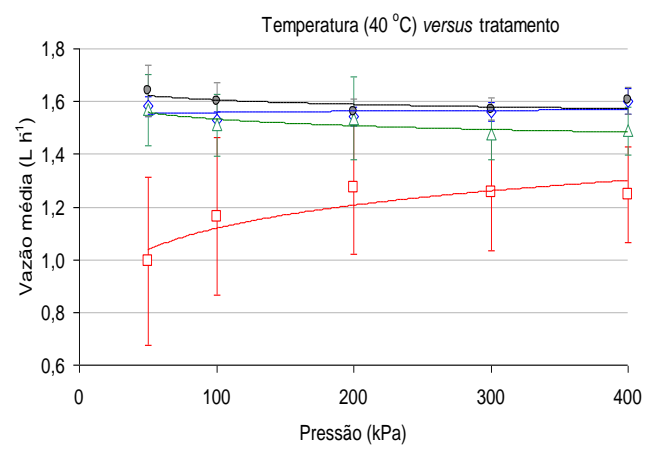

$\diamond \mathrm{D}-\mathrm{T} 40(\mathrm{SA})$

$\square$ D - T40 (100 AD)

$\triangle \mathrm{D}-\mathrm{T} 40(100 \mathrm{AE})$

O D - T40 (pH 2,0 AE)

Figura 1. Curva vazão versus pressão para o modelo D comparando os diferentes tratamentos químicos da água com temperatura da água igual a 15,25 e $40^{\circ} \mathrm{C}$.

O modelo $\mathrm{E}$ apresentou uma redução significativa da vazão (> 80\%), nas temperaturas de 15,25 e $40{ }^{\circ} \mathrm{C}$ para o tratamento químico com aplicação dinâmica de $100 \mathrm{mg} \mathrm{L}^{-1}$ de cloro livre (100 AD). As curvas vazão em função da pressão para os demais tratamentos foram semelhantes em todas as temperaturas, não reduzindo a vazão dos emissores do modelo $\mathrm{E}$, com exceção do tratamento com aplicação estática de $100 \mathrm{mg} \mathrm{L}^{-1}$ de cloro livre (100 AE), que mostrou diminuição na vazão de até $13 \%$ na temperatura de $25^{\circ} \mathrm{C}$.
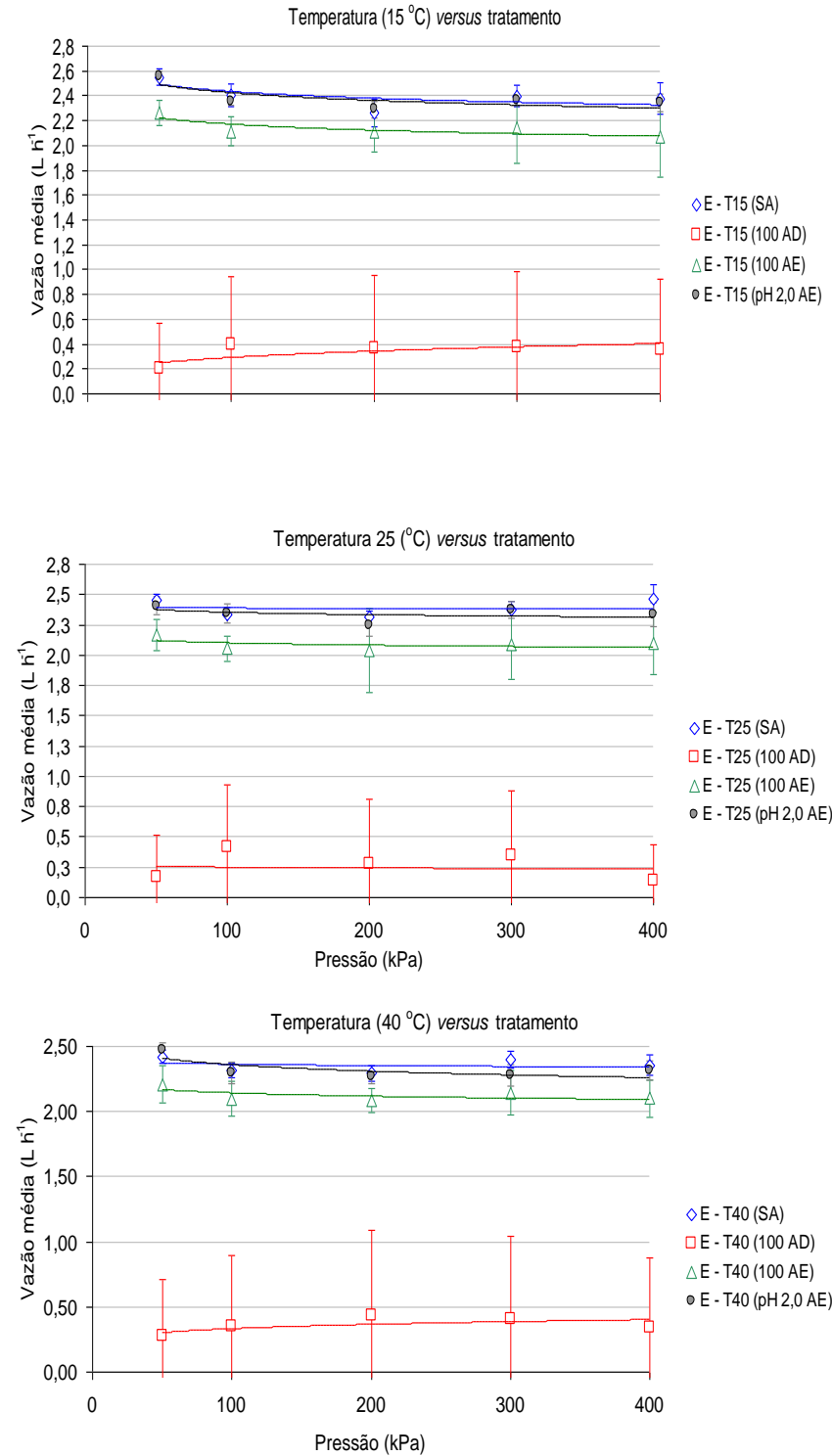

Figura 2. Curva vazão versus pressão para o modelo E comparando os diferentes tratamentos químicos da água com temperatura da água igual a 15,25 e $40^{\circ} \mathrm{C}$.

A redução de vazão mostrada pela curva vazão em função da pressão para o tratamento químico com aplicação dinâmica de $100 \mathrm{mg} \mathrm{L}^{-1}$ de cloro livre (100 AD) (Figura 3), mostrou diferença para o modelo $\mathrm{F}$ em relação aos demais tratamentos em todas as temperaturas. $\mathrm{Na}$ temperatura de $15^{\circ} \mathrm{C}$ o tratamento com aplicação de $100 \mathrm{mg} \mathrm{L}^{-1}$ de cloro livre $(100 \mathrm{AE})$ apresentou vazão superior ao tratamento $\mathrm{SA}$ (5\%) e para a temperatura de $25{ }^{\circ} \mathrm{C}$ e $40{ }^{\circ} \mathrm{C}$ os tratamentos $100 \mathrm{AE} \mathrm{e} \mathrm{pH} \mathrm{2,0} \mathrm{AE} \mathrm{apresentaram}$ vazão superior em todas as pressões em relação ao tratamento SA (6 e 4\%). 

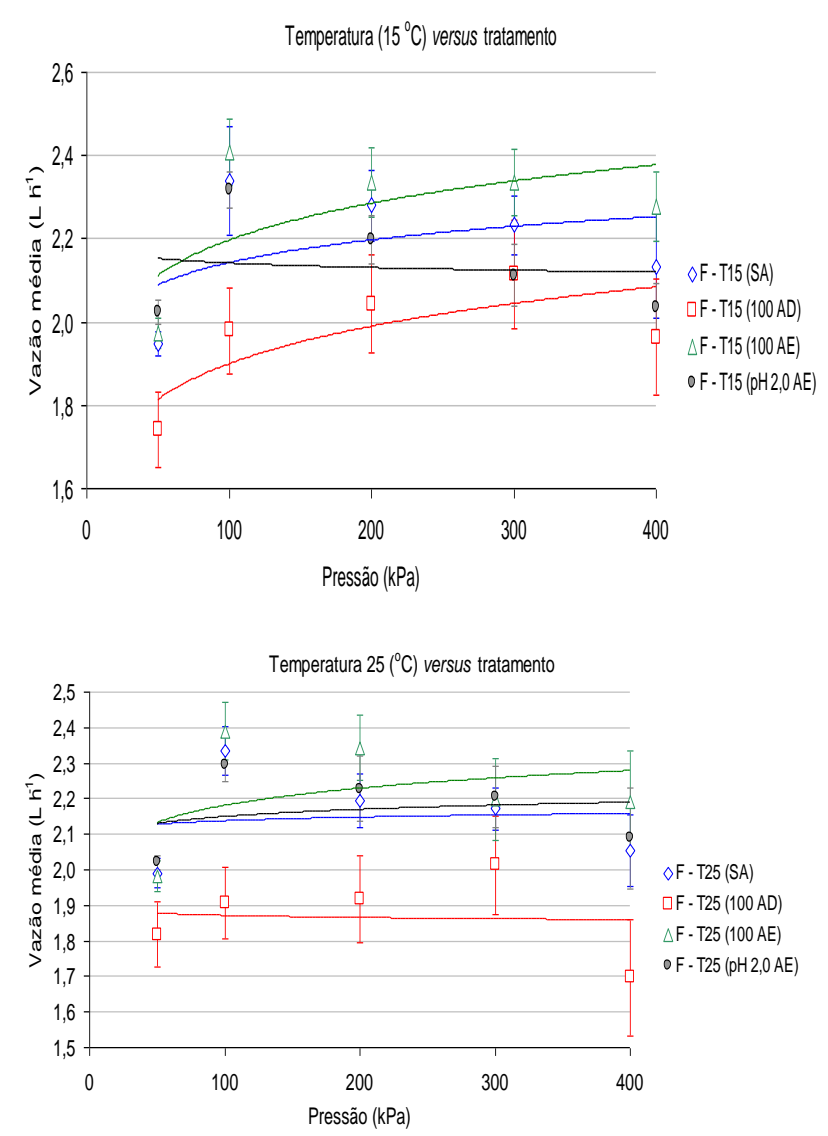

$\diamond \mathrm{F}-\mathrm{T} 25(\mathrm{SA})$

$\square F-T 25(100 \mathrm{AD})$

$\triangle F-T 25(100 \mathrm{AE})$

OF- T25 (pH 2,0 AE)

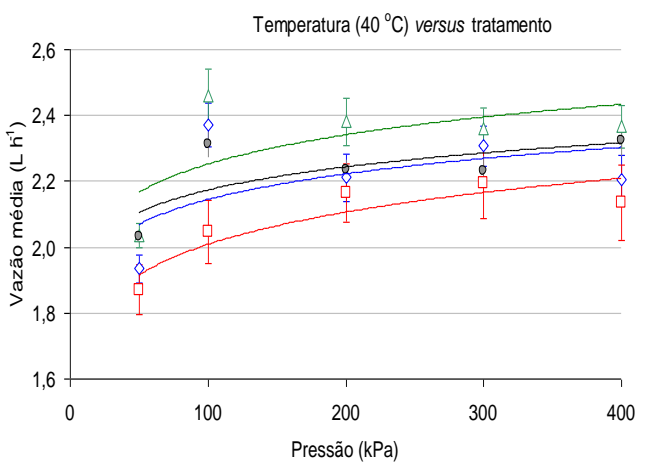

$\diamond \mathrm{F}-\mathrm{T} 40(\mathrm{SA})$

$\square F-T 40(100 \mathrm{AD})$

OF- T40 (pH 2,0 AE)

Figura 3. Curva vazão versus pressão para o modelo F comparando os diferentes tratamentos químicos da água com temperatura da água igual a 15,25 e $40^{\circ} \mathrm{C}$.

O tratamento com aplicação dinâmica de $100 \mathrm{mg} \mathrm{L}^{-1}$ de cloro livre (100 AD) reduziu a zero a vazão do emissor I após 2688 h de funcionamento do sistema, demonstrando a alta susceptibilidade deste modelo a aplicação dinâmica de $100 \mathrm{mg} \mathrm{L}^{-1}$ de cloro livre, logo esse tratamento levou os emissores ao entupimento total de forma drástica e irremediável, pois não indicou vazão nem com o aumento da pressão ou da temperatura, o mesmo foi observado no tratamento 100AE na pressão de $50 \mathrm{kPa}$ em todas as temperaturas. $\mathrm{O}$ tratamento com aplicação estática de $100 \mathrm{mg} \mathrm{L}^{-1}$ de cloro livre (100 AE) diferiu dos tratamentos SA e pH 2,0 AE, apresentando valores de vazão média inferiores principalmente nos pontos de pressões menores $(<300 \mathrm{kPa})$, por conseguinte na pressão de $400 \mathrm{kPa}$ não foi verificado diferenças em relação a vazão para todas as temperaturas.
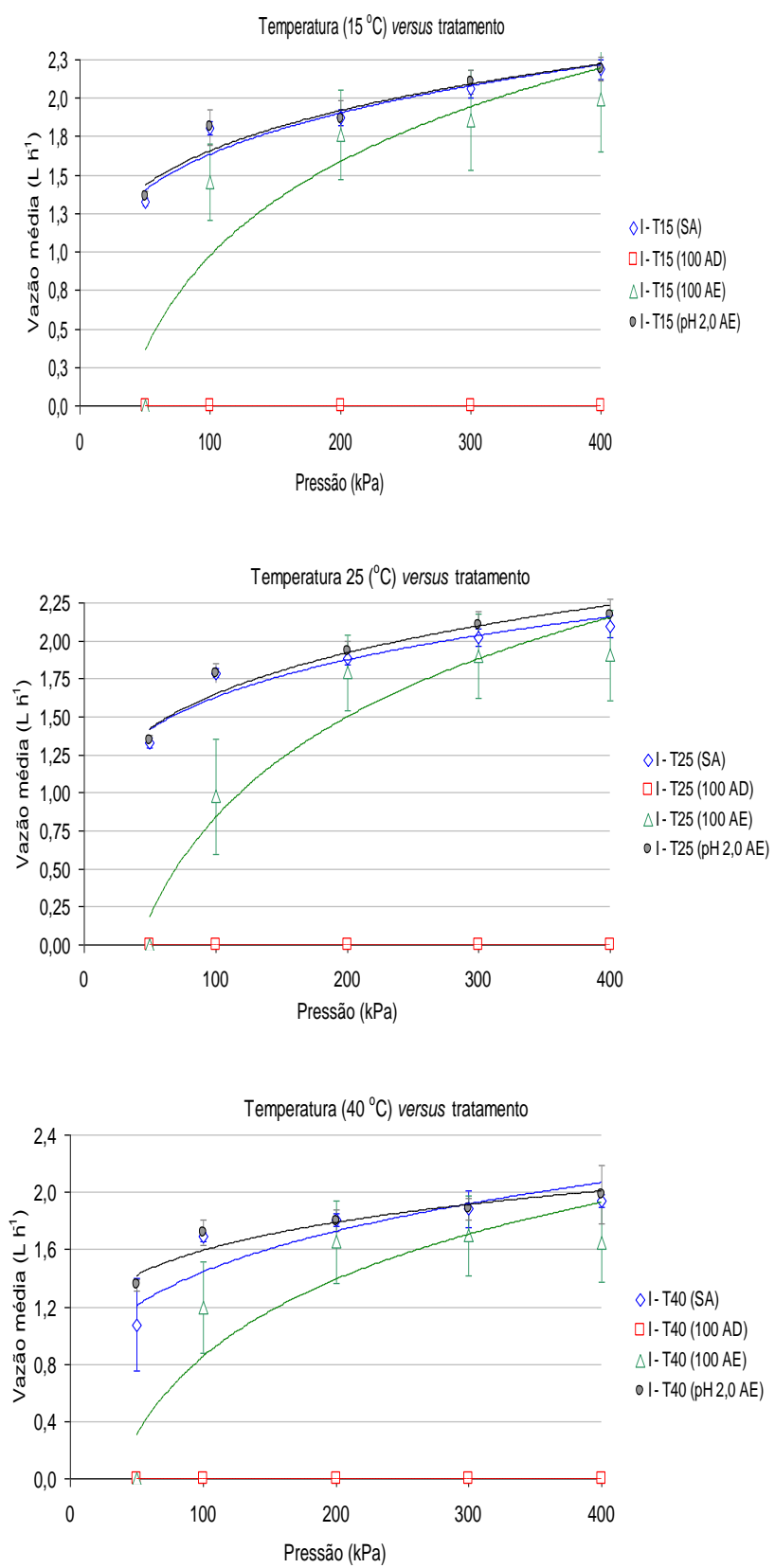

Figura 4. Curva vazão versus pressão para o modelo I comparando os diferentes tratamentos químicos da água com temperatura da água igual a 15,25 e $40^{\circ} \mathrm{C}$. 
Para o emissor $\mathbf{J}$ apenas a curva vazãopressão com aplicação dinâmica de $100 \mathrm{mg} \mathrm{L}^{-1}$ de cloro livre (100 AD) apresentou valores de vazão inferior em todos os pontos de pressão, sendo mais acentuada na temperatura de $25^{\circ} \mathrm{C}$, ficando assim essas diferenças entre 4 e $20 \%$, isto porque este tratamento foi mais agressivo à membrana de compensação do emissor. O mesmo comportamento foi observado para o emissor do modelo $\mathrm{K}$, entretanto o aumento da pressão de maneira geral, nas temperaturas de 15 e $25^{\circ} \mathrm{C}$, tendeu a diminuir a vazão.
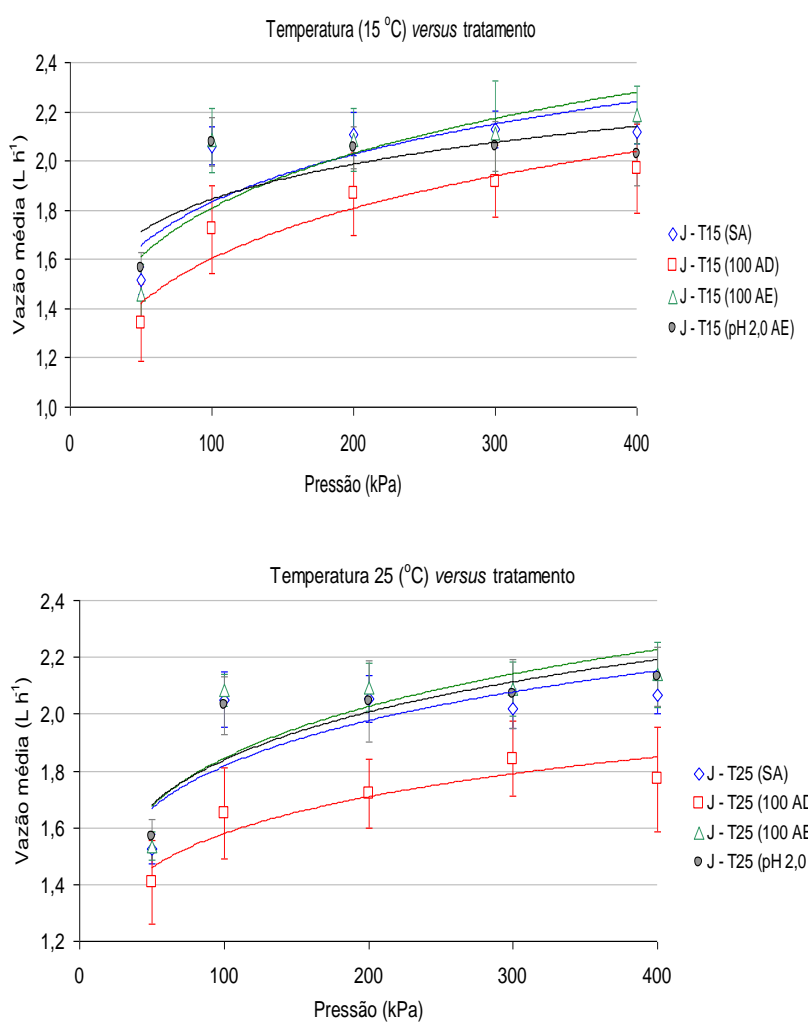

$\triangle \mathrm{J}-\mathrm{T} 25(\mathrm{SA})$

$\square$ J - T25 (100 AD) $\triangle \mathrm{J}-\mathrm{T} 25(100 \mathrm{AE})$ O J - $\mathrm{T} 25(\mathrm{pH} 2,0 \mathrm{AE})$

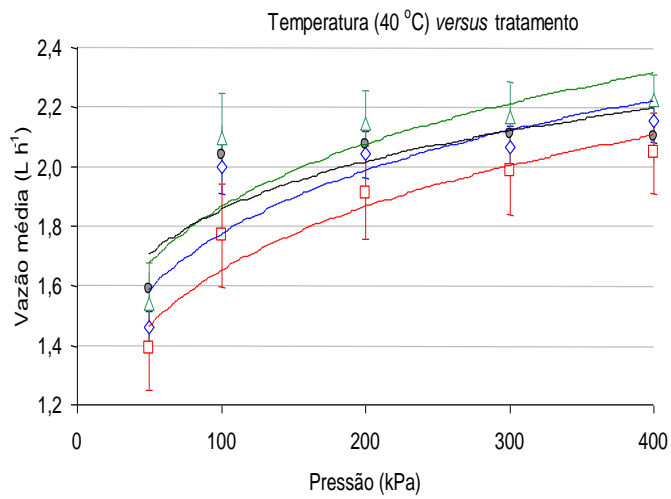

$\diamond \mathrm{J}-\mathrm{T} 40(\mathrm{SA})$ $\square J-T 40$ (100 AD) $\triangle \mathrm{J}-\mathrm{T} 40$ (100 AE) OJ- T40 (pH2,0 AE)

Figura 5. Curva vazão versus pressão para o modelo J comparando os diferentes tratamentos químicos da água com temperatura da água igual a 15,25 e $40^{\circ} \mathrm{C}$.
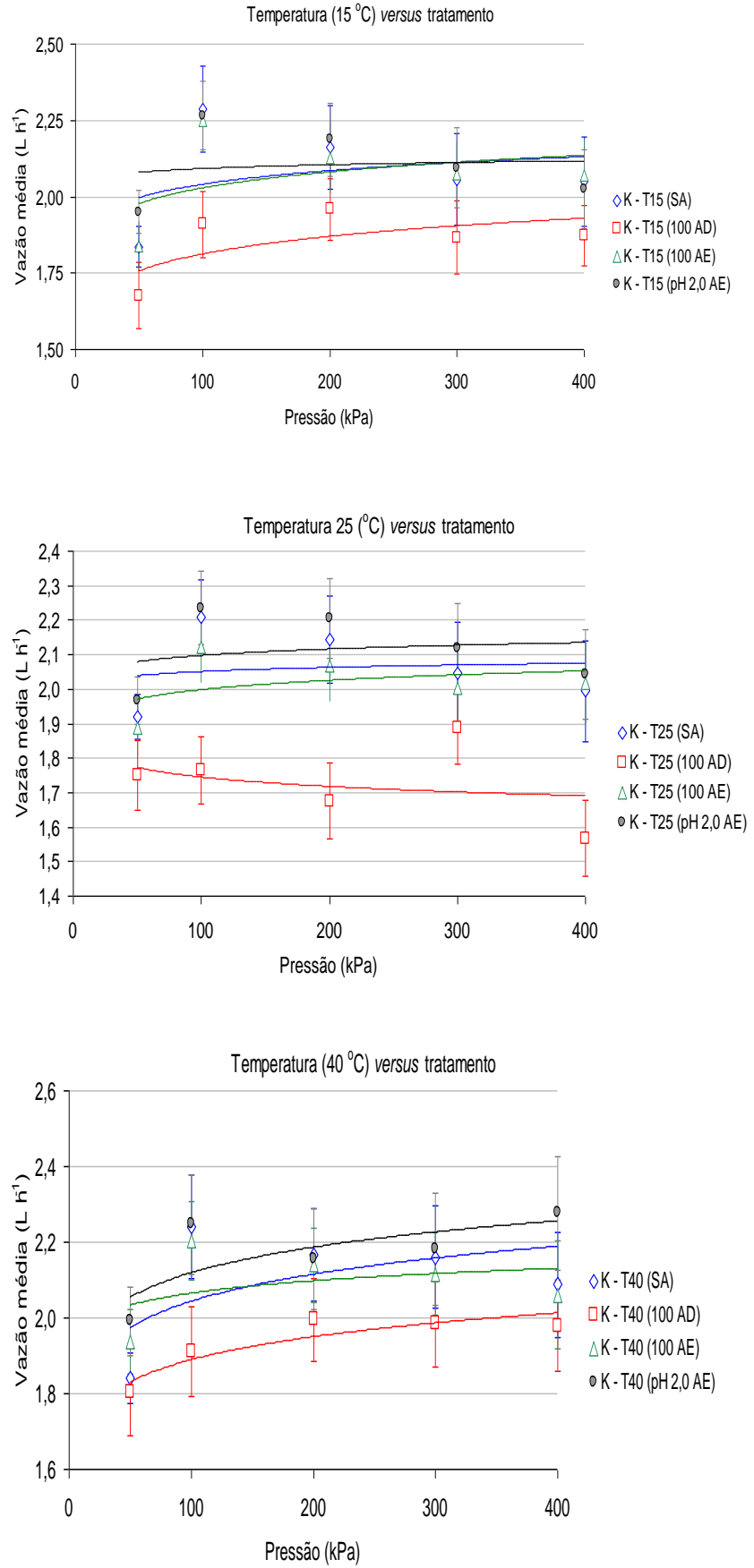

Figura 6. Curva vazão versus pressão para o modelo $\mathrm{K}$ comparando os diferentes tratamentos químicos da água com temperatura da água igual a 15,25 e $40^{\circ} \mathrm{C}$.

A redução de vazão mostrada pela curva vazão-pressão para o tratamento químico com aplicação dinâmica de $100 \mathrm{mg} \mathrm{L}^{-1}$ de cloro livre (100 AD), mostrou a maior diferença de 26,30 e $22 \%$ nas temperaturas de 15,25 e $40^{\circ} \mathrm{C}$ respectivamente, para o modelo $\mathrm{L}$ na pressão de 
$100 \mathrm{kPa}$, em relação aos demais tratamentos.

Para os demais tratamentos não ocorreu diferença entre as curvas vazão-pressão nas diferentes temperaturas.
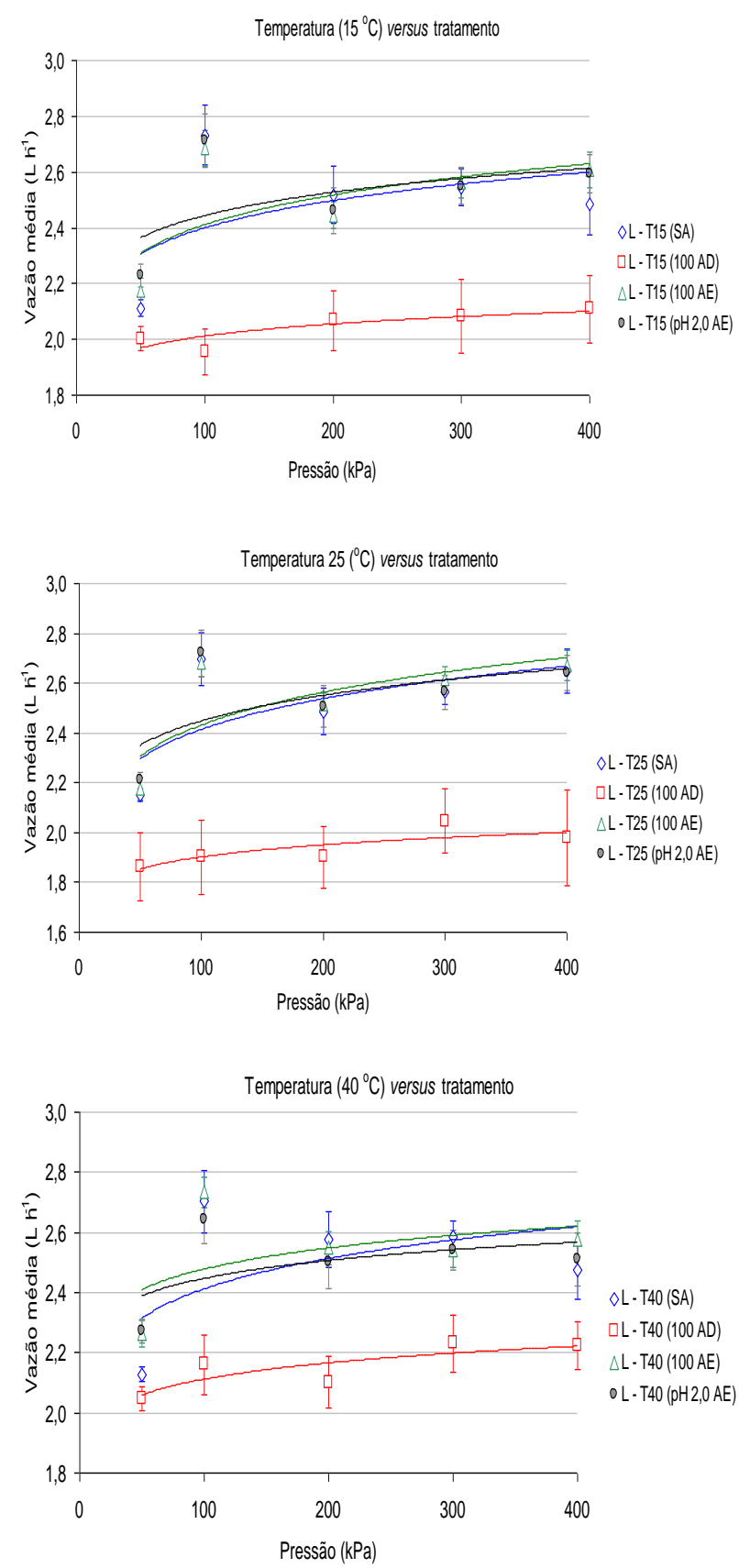

Figura 7. Curva vazão versus pressão para o modelo L comparando os diferentes tratamentos químicos da água com temperatura da água igual a 15,25 e $40^{\circ} \mathrm{C}$.
Observou-se que para todos os emissores o tratamento com aplicação dinâmica de 100 mg L' ${ }^{1}$ de cloro livre (100 AD) foi o que apresentou as maiores reduções de vazão e afetou o comportamento da curva vazão versus pressão nas diferentes temperaturas. Estes resultados estão de acordo com os encontrados por Souza et al. (2006) que utilizando doses de hipoclorito de sódio e diferentes números de aplicações na recuperação da vazão nominal dos gotejadores encontrou resultados favoráveis com aplicação de hipoclorito de até $150 \mathrm{mg} \mathrm{L}^{-1}$ em quatro aplicações. Porém para Vieira et al. (2004) o tratamento com $25 \mathrm{mg} \mathrm{L}^{-1}$ de hipoclorito de sódio foi o que apresentou a melhor relação benefício/custo, sendo o mais econômico e o segundo melhor quanto a uniformidade de irrigação do sistema, o que o torna o mais indicado para limpeza de sistemas de irrigação por gotejamento. Isso mostra que este tratamento deve ser utilizado com restrição quanto ao tempo de contato com a membrana de compensação, conforme a análise comparativa com os tratamentos com aplicação estática de $100 \mathrm{mg} \mathrm{L}^{-1}$ de cloro livre (100 AE - aplicação por apenas $1 / 2 \mathrm{~h}$ da solução), aplicação estática de ácido nítrico por $1 / 2 \mathrm{~h}$ e aplicação estática de ácido nítrico por $12 \mathrm{~h}$. Teixeira et al. (2008) utilizando ácido nítrico a $\mathrm{pH} 2,0$ e $10 \mathrm{mg} \mathrm{L}^{-1}$ de cloro livre, observaram diferença estatística a 5\% de significância pelo 
teste de Tukey em relação ao tratamento que utilizou $50 \mathrm{mg} \mathrm{L}^{-1}$ de cloro livre para desentupimento dos gotejadores. Porém o tratamento com $50 \mathrm{mg} \mathrm{L}^{-1}$ de cloro livre foi o mais eficiente para recuperação da vazão dos gotejadores a valores próximos da vazão nominal.

\section{CONCLUSÕES}

Os emissores podem ser classificados quanto à sensibilidade a ação do cloro como: modelos muito sensíveis (E e I), medianamente sensíveis (D e G), sensíveis (F, J, K e L) e pouco sensíveis (A, B, C, H, M e N);

A análise comparativa entre os diferentes tratamentos químicos utilizados, mostrou que a aplicação dinâmica de $100 \mathrm{mg} \mathrm{L}^{-1}$ de cloro livre foi a que apresentou as maiores reduções de vazão e afetou o comportamento da curva vazãopressão em diferentes temperaturas.

\section{REFERÊNCIAS BIBLIOGRÁFICAS}

BERNARDO, S.; SOARES, A. A.; MANTOVANI, E. C. Manual de irrigação. 8. ed. Viçosa: UFV. 2006, 625p.
CUNHA, F. N.; OLIVEIRA, R. C.; SILVA, N. F.; MOURA, L. M. F.; TEIXEIRA, M. B.; GOMES FILHO, R. R. Variabilidade temporal da uniformidade de distribuição em sistema de gotejamento. Revista Brasileira de Agricultura Irrigada, v.7, n. 4, p. 248 - 257, 2013.

NAKAYAMA, F. S.; BUCKS, D. A. Water quality in drip/trickle irrigation: A review. Irrigation Science, New York, v. 12, p. 187192, 1991.

SOUZA, J. A. A. de; CORDEIRO, E. de A.; COSTA, E. L. da. Aplicação de hipoclorito de sódio para recuperação de gotejadores entupidos em irrigação com água ferruginosa. Revista Brasileira de Engenharia Agrícola e Ambiental, Campina Grande, v. 10, n.1, p. 5-9, 2006.

STATISTICAL ANALYSES SYSTEM - SAS. The SAS system for Windows Version 8. $5^{\text {th }}$ ed. Cary: 1999.

TEIXEIRA, M. B.; MELO, R. F.; COELHO, R. D.; RETTORE NETO, O.; RIBEIRO, P. A. A. Tratamento para desentupimento de gotejadores convencionais. Irriga, Botucatu, v. 13, n. 2, p. 235-248, 2008.

VIEIRA, G. H. S.; MANTOVANI, E. C.; SILVA, J. G. F.; RAMOS, M. M.; SILVA, C. M. Recuperação de gotejadores obstruídos devido à utilização de águas ferruginosas. Revista Brasileira de Engenharia Agrícola e Ambiental, Campina Grande, v. 8, n. 1, p. 1-6, 2004. 